\title{
A Case of Jugular Bulb Diverticulum Invading the Internal Auditory Canal
}

\author{
Jae Hong Park, Seung Beom Son, Hyun Pyo Hong and Hyun-Seok Lee \\ Department of Otorhinolaryngology-Head and Neck Surgery, Bundang Jesaeng Hospital, Daejin Medical Center, Seongnam, Korea
}

Received January 20, 2012

Revised March 13, 2012

Accepted March 17, 2012

\author{
Address for correspondence \\ Hyun-Seok Lee, MD, PhD \\ Department of Otorhinolaryngology- \\ Head and Neck Surgery, \\ Bundang Jesaeng Hospital, \\ Daejin Medical Center, \\ 255-2 Seohyeon-dong, Bundang-gu, \\ Seongnam 463-774, Korea \\ Tel $+82-31-779-0258$ \\ Fax +82-31-779-0265 \\ E-mail dochyun@dmc.or.kr
}

Jugular bulb diverticulum (JBD) is a rarely reported vascular anomaly, which is an extraluminal outpouching from the jugular bulb. Especially, there exists a lack of reported cases involving JBD encroaching the internal auditory canal (IAC) in Korea. Subjects with JBD may be asymptomatic or have variable symptoms based on its location and size. In this article, we report a unique case of JBD eroding into the IAC that was presented as sudden sensorineural hearing loss with vertigo.

Korean J Audiol 2012;16:39-42

\section{Introduction}

The jugular bulb diverticulum (JBD) is an irregular outpouching of the jugular bulb that may extend to the superior surface of the petrous bone, middle ear cavity, endolymphatic duct or vestibular aqueduct. ${ }^{1-3)}$

Subjects with JBD may be asymptomatic or have variable symptoms based on its location and size. JBD may cause conductive hearing loss and pulsatile tinnitus by protruding into the middle ear, and also sensorineural hearing loss (SNHL), as well as vertigo by encroaching the inner ear or the internal auditory canal (IAC). ${ }^{1)}$

JBD is reported rarely, and especially there have been no reported cases involving JBD encroaching the IAC in Korea.

A 54-year-old female with pulsatile tinnitus, vertigo and SNHL was diagnosed with JBD invading the posterior wall of the left IAC. In this article, we report a unique case that is managed with conservative method for JBD invading the IAC.

\section{Case Report}

A 54-year-old female presented with acute onset of severe whirling-type vertigo. The vertigo developed abruptly when the patient got out of the car and aggravated with positional change. The patient also complained of left pulsatile tinnitus like a "snoring" sound. Nausea and vomiting were accompanied initially, however, disappeared within 3 days. There were 5 episodes of vertigo, during the last 5 years. The vertigo was associated with some mental/physical exhaustion and the patient recovered in several days with no medical treatment. The patient denied otalgia, otorrhea and hearing loss.

The patient did not report any specific medical or surgical history but hyperlipidemia. The otoscopic and neurologic exams were unremarkable.

Spontaneous horizontal nystagmus was observed toward the left side at first and changed toward the right side on the fifth hospital day. Pure-tone audiometry on the third hospital day showed SNHL of about $60 \mathrm{dBHL}$ with descending type over $1 \mathrm{kHz}$ and speech discrimination was $28 \%$ on the left side (Fig. 1). Vestibular evoked myogenic potential (VEMP) testing was performed on the fifth hospital day and showed to be normal. Saccade test, pursuit test and optokinetic test presented normal ranges, but caloric test showed the left canal paresis of $21 \%$ on the third hospital day and $73 \%$ on the eighth hospital day. 
Magnetic resonance imaging (MRI) was ordered to rule out the possibility of the left cerebellopontine angle (CPA) tumor. The official report did not identify any abnormal finding. However, we suspected high jugular bulb encroaching the IAC on the left side (Fig. 2). Computed tomography (CT) of the tem-

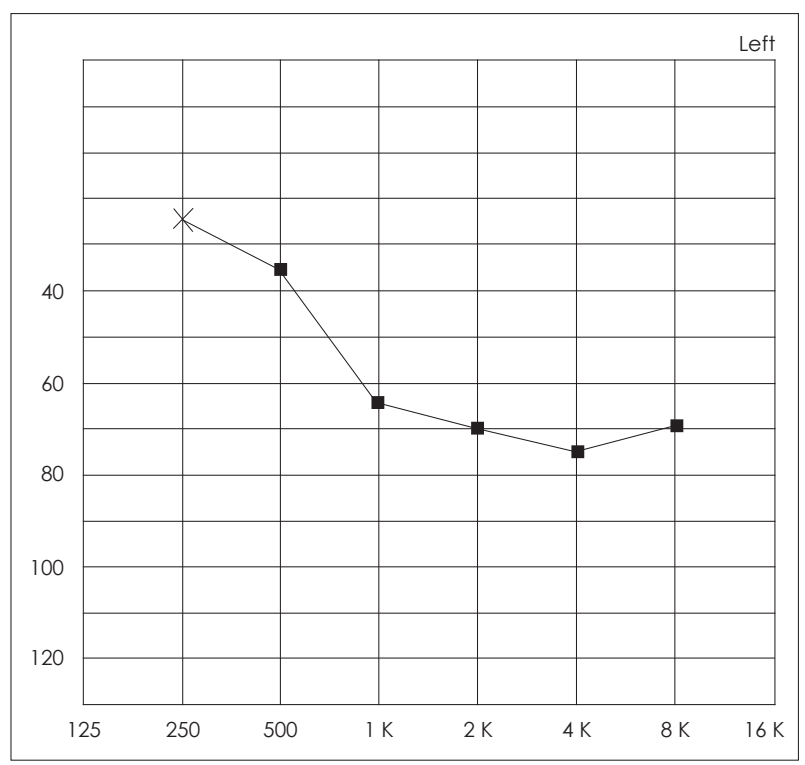

Fig. 1. Initial pure-tone audiogram shows the left high-frequency sensorineural hearing loss. poral bone revealed high jugular bulb and jugular bulb diverticulum with the eroded IAC on the left side (Fig. 3).

When the left high-frequency SNHL was found on the third hospital day, we started to administer $5 \mathrm{mg}$ dexamethasone, intravenously, three times a day, in a regimen of management for sudden sensorineural hearing loss (SSNHL). Even after JBD was diagnosed by radiologic evaluations, the patient received high-dose steroids with the forementioned method for 9 days and underwent a 5-day oral taper, expecting the anti-inflammatory effect of corticosteroids for the cochlear/vestibular nerve.

On the twenty-fifth hospital day, pure-tone audiometry showed high-frequency SNHL with partially recovered threshold of $40 \mathrm{dBHL}$ and speech discrimination score was $72 \%$ on the left side. In a month after initial presentation, the patient still had positional vertigo and nystagmus, with newly developed benign positional paroxysmal vertigo and recovered completely in several days with repositioning maneuvers. Over 21 months after the onset, there has been no recurrence of vertigo, tinnitus or aggravated hearing deterioration.

\section{Discussion}

JBD is considered to be a rare venous anomaly, when com-
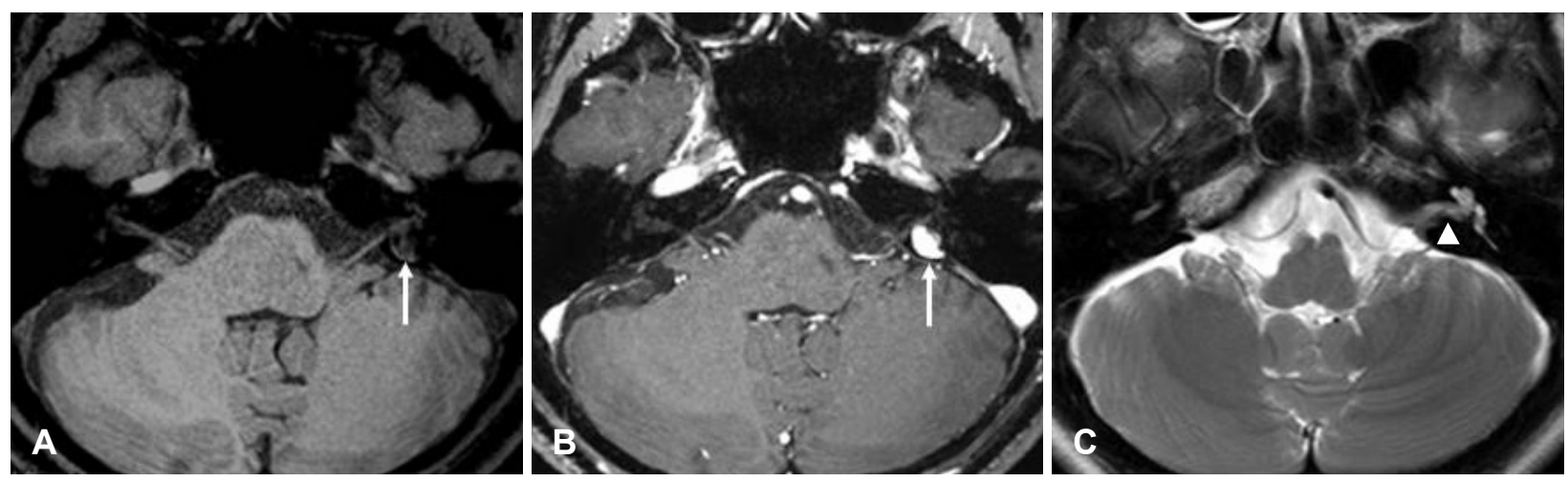

Fig. 2. Internal auditory canal (IAC) magnetic resonance imaging. A: Pre-contrast T1-weighted axial image shows outpouching of the left jugular bulb (arrow). B: Gd-enhanced T1-weighted axial image shows a left jugular bulb compressing the bundle of auditory nerve (arrow). C: Pre-contrast T2-weighted axial image shows a hypointense signal in the left IAC (arrowhead).
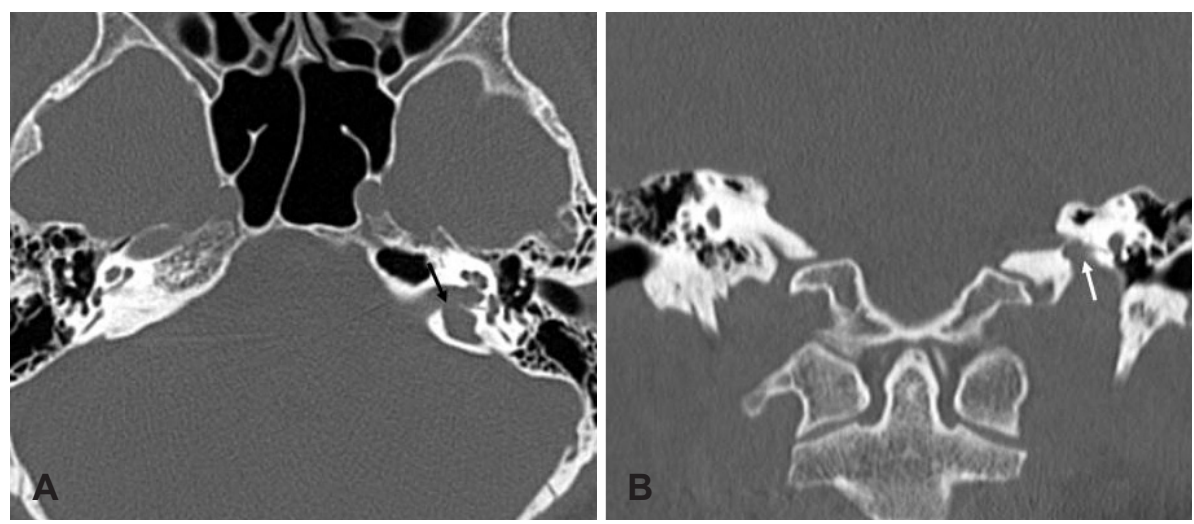

Fig. 3. Computed tomography (CT) of temporal bone reveals a jugular bulb diverticulum (JBD) and erosion of the internal auditory canal (IAC) on the left side. A: Axial CT image shows the eroded posterior wall of the left IAC (black arrow). B: Coronal CT image shows JBD extending to the left IAC (white arrow). 
pared with high-riding jugular bulb that is relatively common venous variation. Retrospective studies, which were performed with $\mathrm{CT}$ of the temporal bone, reported high-riding jugular bulb and JBD ranged from $6 \%$ to $20 \%$ and from less than $1 \%$ to $7.9 \%$, respectively. ${ }^{4,5)}$ However, the rarity of JBD is questionable. Because JBD was rarely reported before high resolution CT (HRCT) and usually discovered at the time of the surgery or as an incidental finding on CT examination of the temporal bone. Furthermore, a significant number of this disease is likely to be asymptomatic, and it is considered that JBD is probably underreported. ${ }^{6}$ )

The etiology of JBD is unrevealed. Embryologically, jugular bulb is not present at birth, but once an erect posture is assumed, as opposed to fetal or lying down position in utero and as neonates, the pounding effects of ascending negative pulse waves originating from the right atrium may traverse upward to the jugular sinus hemodynamically, which leads to an expansion and a formation of the jugular bulb. ${ }^{7)}$ Due to the location of jugular bulb, which is in part, determined by mastoid pneumatization, the distance from the external auditory canal to the sigmoid sinus is relatively shorter in case of little or absent pneumatization. Consequently, jugular bulb rides relatively high, which is also a risk factor for JBD. ${ }^{8)}$ Though venous hypertension and turbulent venous flow have been suggested as an etiologic factor for venous diverticulum with bony erosion, a definitive understanding of the pathophysiology of diverticulum formation remains elusive.

Depending on the degree of extension of JBD, the cochlear and vestibular aqueducts, the endolymphatic sac, the posterior semicircular canal, the mastoid cavity and IAC can be involved. ${ }^{1-3)}$ Therefore, various symptoms, which includes SNHL, vertigo, aural fullness and tinnitus, can be produced in accordance with the location and size of JBD.

A few cases of JBD encroaching IAC have been reported, however most of cases were identified in the retrospective studies. ${ }^{2,3)}$ Thus, their clinical manifestations were not described in detail. Fujimoto, et al. ${ }^{9)}$ reported one case of JBD invading IAC in detail, however, clinical presentation was much different from our case. In his study, the patient had a conductive hearing loss especially at the low tone and subclinical vestibular dysfunction without vertigo, despite the similarity with our study in regards to the imaging study of the patient.

In our case, CT images showed JBD encroaching only the posterior wall of the left IAC without the involvement of the endolymphatic sac, the vestibular aqueduct or the posterior semicircular canal. It is considered that the audiologic and vestibular symptoms, such as pulsatile tinnitus, high frequency sensorineural hearing loss and vertigo resulted from the compression and irritation of cochleovestibular nerve, caused by JBD. However, hemifacial spasm didn't develop because the diverticulum eroded the IAC from below, as well as from behind, and anatomically the facial nerve was spared. When the symptoms started, spontaneous nystagmus toward the left side had been observed, but in three days, it changed toward the right direction. Also, left canal paresis was aggravated from $21 \%$ weakness on the third hospital day to $73 \%$ weakness on the eighth hospital day, according to the serial caloric tests. It is assumed that the left vestibular nerve was irritated by the diverticulum at the initial state. It might get paralytic as neurovascular cross-compression persisted.

JBD can be identified mostly by radiologic evaluation, including CT or MRI. However, when a patient has unilateral cochlear symptoms, with or without vertigo, different diseases, such as SSNHL, Meniere's disease, superior canal dehiscence syndrome, CPA tumor should be considered, and MRI is generally chosen as the imaging method. However, we could not easily detect small venous anomalies, such as JBD without intensive suspicion. HRCT is the method of choice when jugular bulb anomalies are suspected. ${ }^{1,6)}$ Bush, et al. ${ }^{3)}$ suggested that the CT venography should be used in the evaluation of asymmetric audiologic symptoms, and vertigo in case of negative MRI findings. Further, they said that CT venography can be the primary study in patients with pulsatile tinnitus alone. In our case, since the patient had high-frequency hearing loss with poor speech discrimination and vertigo, MRI should be checked prior to ruling out CPA tumor.

VEMP testing provides diagnostic information about saccular and/or inferior vestibular nerve function. VEMP was normal in this case. Although it is assumed that the functional deficit of inferior vestibular nerve and posterior semicircular canal was little or absent in that time of examination, it doesn't exclude subclinical or progressive functional deficit. Therefore, periodic and close observation should be followed.

Because little is known about the natural course of JBD, guidelines for treament are not established in detail. Consequently, it is difficult and complicated in a part to decide whether we should treat conservatively or surgically. According to a few literatures, since JBD is not static and enlarges with time, it can gradually aggravate patient's symptoms. ${ }^{10)}$ Further, another author said pulsatile tinnitus that is caused by highriding jugular bulb or JBD can be diminished and even disappear naturally. ${ }^{11,12)}$ In our case, pulsatile tinnitus started to diminish in a few days and disappeared in a week, and vertigo improved gradually. Therefore, we decided to manage conservatively with medication, expecting anti-inflammatory reaction of corticosteroids.

If patients with severe symptoms of vascular origins, such as annoying tinnitus, continuous vertigo and persistent or pro- 
gressive hearing loss, surgical managements can be considered. But a decision for surgery should be based on careful evaluation of the risks and benefits of each case.

\section{REFERENCES}

1) Friedmann DR, Le BT, Pramanik BK, Lalwani AK. Clinical spectrum of patients with erosion of the inner ear by jugular bulb abnormalities. Laryngoscope 2010;120:365-72.

2) Bilgen C, Kirazli T, Ogut F, Totan S. Jugular bulb diverticula: clinical and radiologic aspects. Otolaryngol Head Neck Surg 2003;128: 382-6.

3) Bush ML, Jones RO, Given C. The value of CT venography in the diagnosis of jugular bulb diverticulum: a series of 3 cases. Ear Nose Throat J 2009;88:E4-7.

4) Atilla S, Akpek S, Uslu S, Ilgit ET, Işik S. Computed tomographic evaluation of surgically significant vascular variations related with the temporal bone. Eur J Radiol 1995;20:52-6.

5) Koesling S, Kunkel P, Schul T. Vascular anomalies, sutures and small canals of the temporal bone on axial CT. Eur J Radiol 2005;54:33543.

6) El-Kashlan HK, Arts HA, Gebarski S. Jugular diverticulum: clinical significance. Otolaryngol Head Neck Surg 2000;122:575-6.

7) Okudera T, Huang YP, Ohta T, Yokota A, Nakamura Y, Maehara F, et al. Development of posterior fossa dural sinuses, emissary veins, and jugular bulb: morphological and radiologic study. AJNR Am J Neuroradiol 1994;15:1871-83.

8) Wadin K, Thomander L, Wilbrand H. Effects of a high jugular fossa and jugular bulb diverticulum on the inner ear. A clinical and radiologic investigation. Acta Radiol Diagn (Stockh) 1986;27:629-36.

9) Fujimoto C, Ito K, Ishimoto S, Iwasaki S. Large jugular bulb diverticulum invading the internal auditory canal. Ann Otol Rhinol Laryngol 2007;116:631-6.

10) Wadin $K$, Wilbrand $H$. The jugular bulb diverticulum. A radioanatomic investigation. Acta Radiol Diagn (Stockh) 1986;27:395-401.

11) Adler JR, Ropper AH. Self-audible venous bruits and high jugular bulb. Arch Neurol 1986;43:257-9.

12) Chandler JR. Diagnosis and cure of venous hum tinnitus. Laryngoscope 1983;93:892-5. 